\title{
HOX Antisense lincRNA HOXA-AS2 Promotes Tumorigenesis of Hepatocellular Carcinoma
}

\author{
Fuqiang Wang ${ }^{\mathrm{a}}$ Huili Yang ${ }^{\mathrm{b}}$ Zhigang Deng $^{\mathrm{c}}$ Yongjie Su ${ }^{\mathrm{a}}$ QinLiang Fang ${ }^{\mathrm{a}}$ \\ Zhenyu Yina
}

aDepartment of Hepatobiliary Surgery, Zhongshan Hospital of Xiamen University, Fujian Provincial Key Laboratory of Chronic Liver Disease and Hepatocellular Carcinoma, Xiamen, Fujian, 'bepartment of neurology, Zhongshan Hospital of Xiamen University, Xiamen, Fujian, 'Department of General Surgery, MianYang Central Hospital, Mianyang Sichuan, China

\section{Key Words}

HOXA-AS2 • Hepatocellular carcinoma $•$ Cell proliferation $•$ Cell migration

\begin{abstract}
Background: Recent studies reveal that long non-coding RNAs (LncRNAs) play critical roles in the proliferation and migration of human cancer. Previous report has shown that LncRNA HOXA-AS2 was involved in carcinoma processes. However, the expression and biological function of HOXA-AS2 in hepatocellular carcinoma (HCC) are poorly understood. Methods: Quantitative real-time PCR (qRT-PCR) was performed to detect the expression of HOXAAS2 in HCC tissues and cell lines. The relation between IncRNA HOXA-AS2 expression and clinicopathological characteristics was assessed by chi-square test. The prognosis was analyzed using Kaplan-Meier method, and compared differences between the two groups by log-rank test. The biological function of HOXA-AS2 on HCC cells were determined both in vitro and in vivo. Results: In the present study, we found that HOXA-AS2 expression was increased in HCC tissues and adjacent normal tissues and high HOXA-AS2 expression was associated with bigger tumor size, advanced tumor stage, and shorter survival time. Knockdown of HOXAAS2 significantly inhibited HCC cell proliferation and invasion and resulted in an increase of apoptosis. Furthermore, inhibition of HOXA-AS2 in HCC cells significantly repressed tumorigenicity in nude mice. Conclusion: Our results indicated that the inhibition of HOXAAS2 in HCC cells significantly inhibited cell proliferation in vitro and in vivo, which might provide a potential possibility for targeted therapy of HCC.

\section{Introduction}

Hepatocellular carcinoma (HCC) is the fifth most common cancer all over the world especially in East Asia and the third leading cause of cancer-related deaths globally [1, 2].

F. Wang and $\mathrm{H}$. Yang contributed equally. 


\section{Cellular Physiology Cell Physiol Biochem 2016;40:287-296 \begin{tabular}{l|l|l|}
\hline DOI: 10.1159/000452545 & $\begin{array}{l}\text { () 2016 The Author(s). Published by S. Karger AG, Basel } \\
\text { www.karger.com/cpb }\end{array}$ \\
\hline
\end{tabular} \\ Wang et al.: HOXA-AS2 Medicates HCC Proliferation and Migration}

The tumorigenesis and progression of HCC is a complex process influenced by multiple external environment and genetic factors, early diagnosis and intervention is imperative to improve efficacy of treatments. Despite recent improvements in radical surgery and liver transplantation, the prognosis of HCC is poor due to postoperative recurrence or/and metastasis $[3,4]$. Therefore, the further investigation of underlying molecular mechanism and therapeutic targets of HCC is essential for the development of novel effective therapies [4].

Long noncoding RNAs (lncRNAs), which are transcribed by RNA polymerase without protein coding capacity, have recently been shown to play a key role in regulating a wide range of cellular functions including cancer progression [5-9]. Emerging evidence indicated that dysregulation of lncRNAs acted as drivers of oncogenic function or tumor suppressors have been found to affect cell proliferation, apoptosis, differentiation, invasion and metastasis in diverse cancer types [10-12]. For example, IncRNA Linc00974 was found to increase in HCC owing to an abnormal hypomethylation promoter and knockdown of Linc00974 inhibited cell proliferation and invasion in vitro and in vivo [13]. LncRNA HOTAIR functions as an oncogene and high expression level of HOTAIR is correlated with metastases and survival in many cancers [14-18]. HOXA-AS2, a long non-coding RNA located between the HOXA3 and HOXA4 genes in the HOXA cluster, has been characterized as an oncogene in acute promyelocytic leukemia and gastric cancer, and knockdown of HOXA-AS2 inhibited cell proliferation and induced cell apoptosis $[19,20]$. However, whether HOXA-AS2 is involved in the progression of HCC is ill-defined and the molecular mechanisms remain to be fully elucidated.

In this study, we found that HOXA-AS2 was up-regulated in HCC tissues than that in corresponding non-tumor tissues. Furthermore, HOXA-AS2 expression associated with tumor size, TNM stage, and outcome of HCC patients. Additionally, investigate the detailed effects and mechanisms of HOXA-AS2 in HCC, both in vivo and in vitro. Our results shed some light on the role of HOXA-AS2 in the progression of HCC, which could be used as a potential target for the treatment of HCC.

\section{Materials and Methods}

\section{Tissue samples}

112 paired HCC and corresponding adjacent non-tumorous liver samples were collected from patients who underwent surgery at Zhongshan Hospital of Xiamen University between 2008 and 2010. No chemotherapy or radiotherapy was conducted in these patients before surgery. The diagnosis was confirmed as HCC based on haematoxylin and eosin stained sections by an experienced pathologist (Z.X.C). This study was approved by The Clinical Research Ethics Committee of Zhongshan Hospital of Xiamen University. Written informed consent was obtained from all participants enrolled in this study. All the specimens were immediately snap-frozen in liquid nitrogen in liquid nitrogen and stored at $-80^{\circ} \mathrm{C}$ until use.

\section{Cell lines}

The LO2 immortalized human liver cell line and four human HCC cell lines (Huh7, Hep3B, SMMC7721, and BEL-7402) were obtained from the Cellbank of the Chinese Academy of Sciences in Shanghai (Shanghai, China). All cells were cultured in Dulbecco's modified Eagle's medium (Thermo, Beijing, China) supplemented with $10 \%$ fetal bovine serum (Gibco, Carlsbad, CA, USA), $100 \mathrm{U} / \mathrm{ml}$ penicillin and $100 \mathrm{mg} / \mathrm{ml}$ streptomycin in a humidified $5 \% \mathrm{CO}_{2}$ incubator at $37^{\circ} \mathrm{C}$.

Quantitative real time polymerase chain reaction ( $q R T-P C R$ )

Total RNA was isolated from tissues and cells by TRIzol reagent (Invitrogen, Carlsbad, CA, USA) according to the manufacturer's protocol. To analyze HOXA-AS2 expression, first-strand cDNAs were reverse transcribed from total RNA using a Reverse Transcription Kit (Takara, Dalian, China). qRTPCR was performed with SYBR Green PCR Master Mix (Toyobo, Osaka, Japan) on an ABI Prism 7900HT instrument (Applied Biosystems, CA, USA). The forward and reverse HOXA-AS2 primer sequences were 


\section{Cellular Physiology Cell Physiol Biochem 2016;40:287-296 \begin{tabular}{l|l|l}
\hline DOI: 10.1159/000452545 & $\begin{array}{l}\text { C) 2016 The Author(s). Published by S. Karger AG, Basel } \\
\text { www.karger.com/cpb }\end{array}$ \\
\hline Published online: November 18, 2016
\end{tabular} \\ Wang et al.: HOXA-AS2 Medicates HCC Proliferation and Migration}

5'-AACCCATCTTTGCCTTCTGC-3' and 5'-CGGAGGAGTTTGGAGTTGG-3', respectively. GAPDH was used as the internal control. The results were analyzed and expressed relative to threshold cycle (CT) values, and then converted to fold changes.

\section{Gene silencing of HOXA-AS2 in HCC cell lines}

The siRNA duplexes targeting HOXA-AS2 were designed by (Zhongqing, Suzhou, Jiangsu, China) and synthesized by Sangon (ShangHai, China). Cells were treated with negative control or siRNA at a final concentration of 50nM using X-tremeGENE siRNA Transfection Reagent (Roche, Indianapolis, IN, USA) according to the manufacturer's protocols. The knock-down efficiency of the HOXA-AS2 by siRNAs was confirmed by qRT-PCR.

\section{Cell growth and colony formation assay}

Cell viability was assessed with a Cell Proliferation Reagent WST-1 (Roche). Logarithmic growth phase cells were seeded at a density of $2 \times 10^{3}$ cells per well in $100 \mu \mathrm{l}$ of complete medium into 96 -well plates. WST- 1 reagent was added at 0,72 , and $96 \mathrm{~h}$ after seeding and incubated at $37^{\circ} \mathrm{C}$ for $2 \mathrm{~h}$. Cell viability was determined with a microplate reader (BioTek, Winooski, VT, USA) at a wavelength of $450 \mathrm{~nm}$. For colony formation assay, $1 \times 10^{3}$ cells were placed into of 6 -well plates and maintained in complete medium for two weeks, the medium was replaced every 3 days. The cells were fixed with $4 \%$ paraformaldehyde and stained with $0.5 \%$ crystal violet (Sigma-Aldrich, St. Louis, MO, USA). Visible colonies were then manually counted. Each experiment was performed in triplicate and repeated at least three times.

\section{Apoptosis assay}

Cell apoptosis was evaluated using the Annexin V-FITC Apoptosis Detection Kit (BD Biasciences, NJ, USA) according to the manufacturer's instructions. The cells were analyzed by BD Biosciences FACSCalibur Flow Cytometer (BD Biosciences) equipped with a CellQuest software (BD Biosciences).

\section{Migration and Invasion assay}

Transwell assay was performed to assess cell migration and invasion. Transwell inserts with $8 \mu \mathrm{m}$ pore size (Corning Costar, NY, USA) were uncoated with Matrigel or precoated with Matrigel (BD Biosciences) for migration or invasion assay as previously described [21], respectively. In brief, $5 \times 10^{4}$ cells were seeded into upper uncoated inserts for migration assays; $1 \times 10^{5}$ cells were seeded into upper inserts with a Matrigelcoated for invasion assays. After $36 \mathrm{~h}$ of incubation at $37^{\circ} \mathrm{C}$, the cells that had migrated through the filters were fixed, stained and counted from 10 random fields under inverted microscopy at $200 \times$ magnification.

The subcutaneous xenotransplantation model

BALB/c nude mice (4 weeks old) were purchased from the Animal Center of Southern Medical University (Guangzhou, China) and maintained under specific pathogen-free conditions. BEL-7402 cells (1 $\times 10^{7}$ cells) transfected with control or siRNA of HOXA-AS2 were injected subcutaneously into the right axilla of each mouse. Tumor volume was examined by caliper measurement every week and calculated (length $\times$ width $^{2}$ ) $/ 2$. All mice were sacrificed 5 weeks after injection and the tumors were dissected, photographed and weighed. This study was conducted according to the recommendations in the Guide for the Care and Use of Laboratory Animals of the National Institutes of Health and approved by the Committee on the Ethics of Animal Experiments of Xiamen University.

\section{Western blot analysis}

Total protein was extracted cultured cells using RIPA buffer (Cell Signaling, Danvers, MA, USA) containing $1 \mathrm{mM}$ phenyl methane sulfonyl fluoride (PMSF) (Sigma-Aldrich). Protein was separated by $10 \%$ SDS-PAGE and transferred to a polyvinylidene fluoride membrane (Bio-Rad, Hercules, CA, USA), blocked in $5 \%$ nonfat milk for 2 hours at room temperature, and visualized using an ECL kit (Millipore, MA, USA). The following antibodies were used for analysis: anti-c-Myc (Cell Signaling), anti-Bax (Cell Signaling), anti-BCL-2 (Cell Signaling), anti-MMP-2 (Proteintech group, Inc., Chicago, IL, USA), anti-MMP-9 (Proteintech group, Inc., Chicago, IL, USA), anti-p-AKT (Cell Signaling), anti-AKT (Cell Signaling), and anti-GAPDH (Santa Cruz, CA, USA). 
Immunohistochemical (IHC) analysis

The tumors xenografts were immunostained for Ki-67 (Sigma) as previously described [22].

\section{Statistical analysis}

Data were expressed as mean \pm SD of 3 independent experiments. The difference between two groups was determined by the Student's t-test using GraphPad software version 5.0 (GraphPad Software, CA, USA). The Kaplan-Meier method was performed to analyze survival data and the log-rank test was taken to assess differences in patients' survival. P value less than 0.05 was considered statistically significant.

\section{Results}

Expression of HOXA-AS2 is increased in HCC cells and tissues

To investigate whether the expression of HOXA-AS2 was altered in HCC, we measured the expression of HOXA-AS2 in HCC cells by qRT-PCR. As shown in Figure 1A, HOXA-AS2 was increased in all of the 4 analysed HCC cell lines compared with the normal liver cell line (LO2). Subsequently, HOXA-AS2 expression was determined by qRT-PCR in 112 matched HCC and adjacent normal tissues. compared with the adjacent normal tissues, HOXA-AS2 was significantly elevated in HCC samples (Fig. 1B). Furthermore, the expression of HOXAAS2 was positively associated with the TNM stage and remarkably upregulated in TNM IIIII stage. These results suggested that HOXA-AS2 was overexpressed in HCC and might be involved in the progression of HCC.

\section{Upregulated HOXA-AS2 expression predicts a poor prognosis in HCC patients}

We next assess whether the expression of HOXA-AS2 associated with the clinical outcome in patients with HCC. We divided the 112 HCC patients into a high HOXA-AS2 expression group $(n=56)$ and a low HOXA-AS2 expression group $(n=56)$ according to the intermediate values of HOXA-AS2. As shown in Table 1, the expression levels of HOXA-AS2 was associated with the tumor size and TNM stage. No statistical correlation with gender,

Fig. 1. HOXA-AS2 expression in HCC cell lines and HCC tissues. (A) qRT-PCR was performed to determine HOXA-AS2 expression in LO2, Huh7, Hep3B, SMMC-7721 and BEL7402 cells. (B) Relative expression of HOXAAS2 was determined in HCC tissues compared with adjacent non-tumor tissues by qRTPCR. (C) HOXA-AS2 level was categorised according to tumour-nodule-metastasis stage. ** indicated $\mathrm{P}<0.01$.

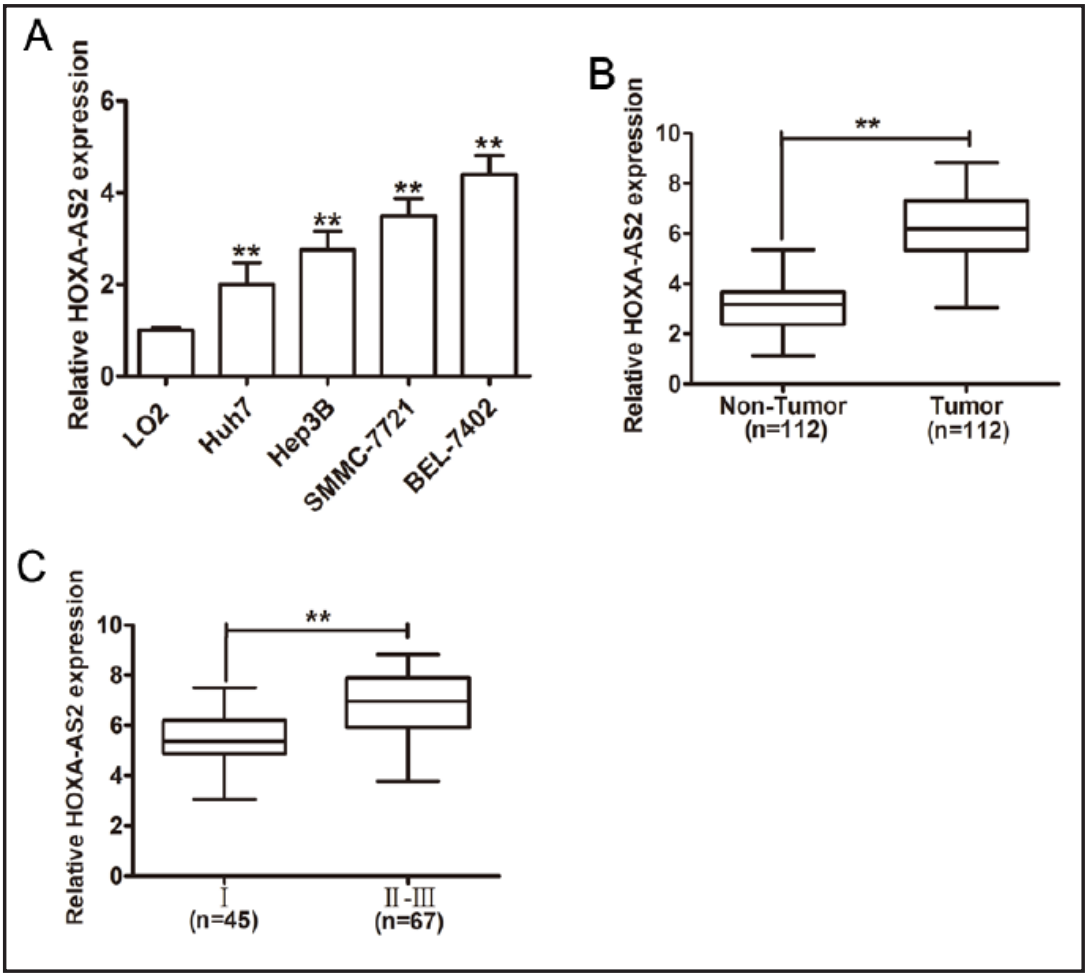


Table 1. Correlation between HOXA-AS2 expression and clinicopathological features of HCC patients $(n=112)$

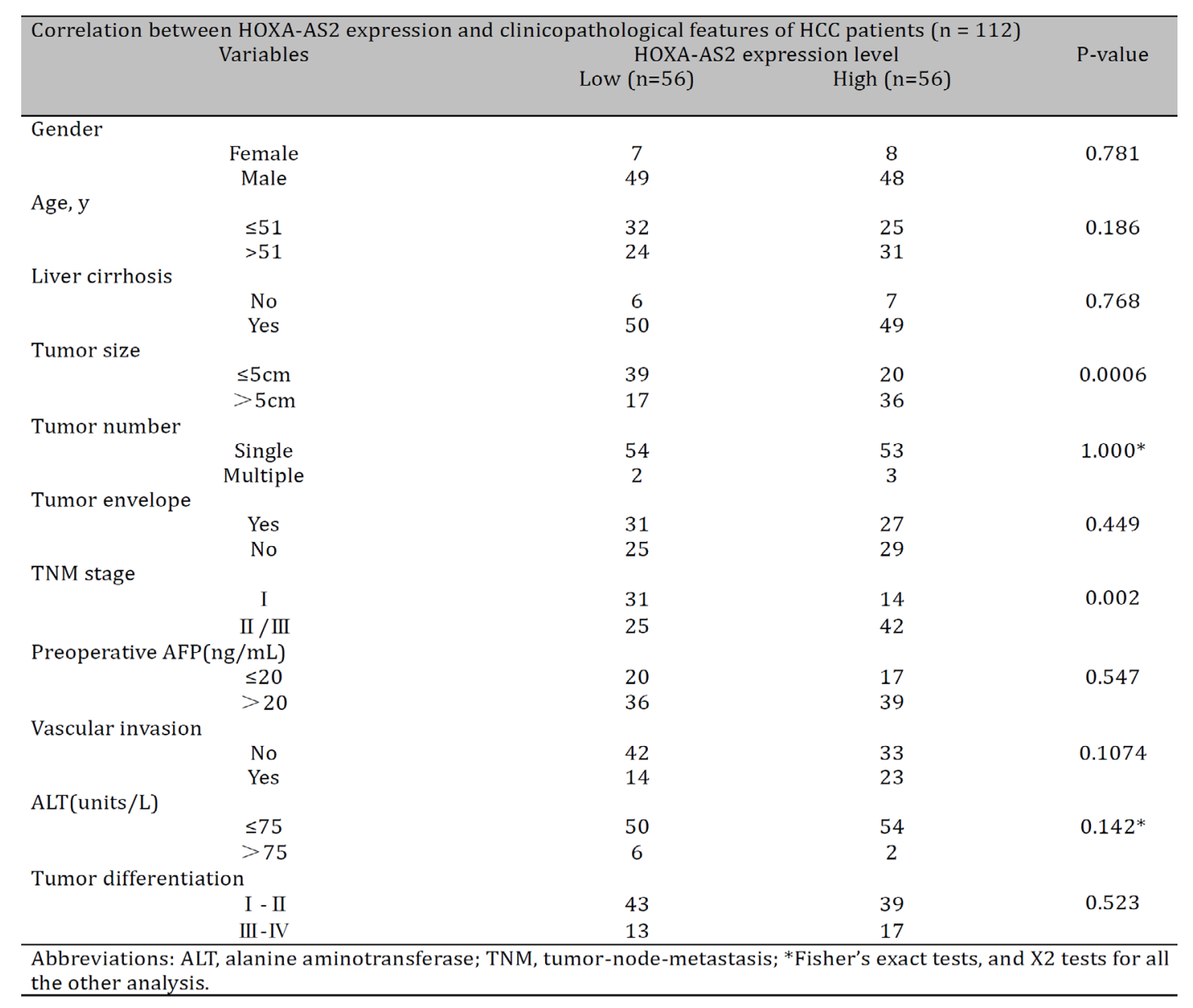

Fig. 2. HOXA-AS2 expression in HCC correlated with tumor prognosis. Kaplan-Meier was applied to assess overall survival (OS) curve of HCC patients with different expressions of HOXA-AS2.

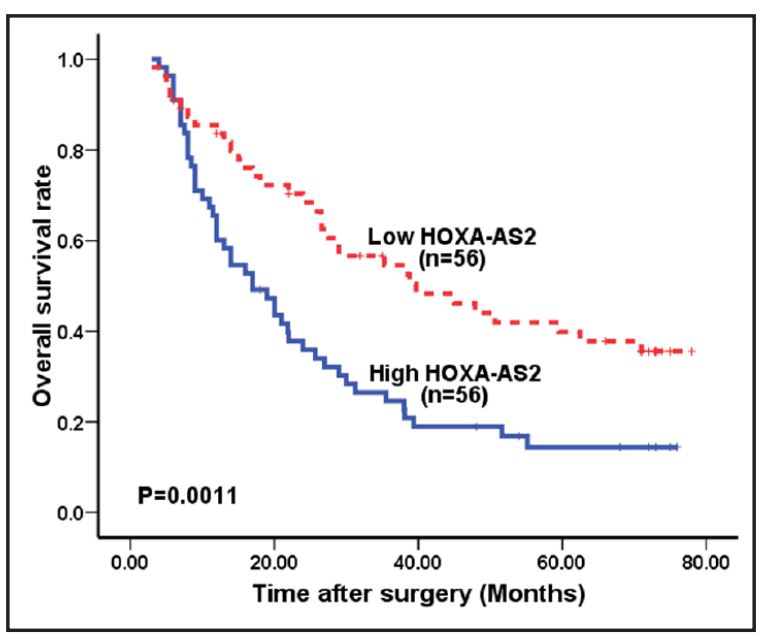

age, liver cirrhosis and tumor number was observed. Furthermore, Kaplan-Meier survival indicated that patients with high HOXA-AS2 expression level had a shorter overall survival time than those of low level (Fig. 2).

Knockdown of HOXA-AS2 attenuates proliferation and clonogenicity of HCC cells

To determine the functional role of HOXA-AS2 in HCC, we knockdown HOXA-AS2 with siRNA in SMMC-7721 and BEL-7402 cells, which have high endogenous HOXA-AS2 expression. 


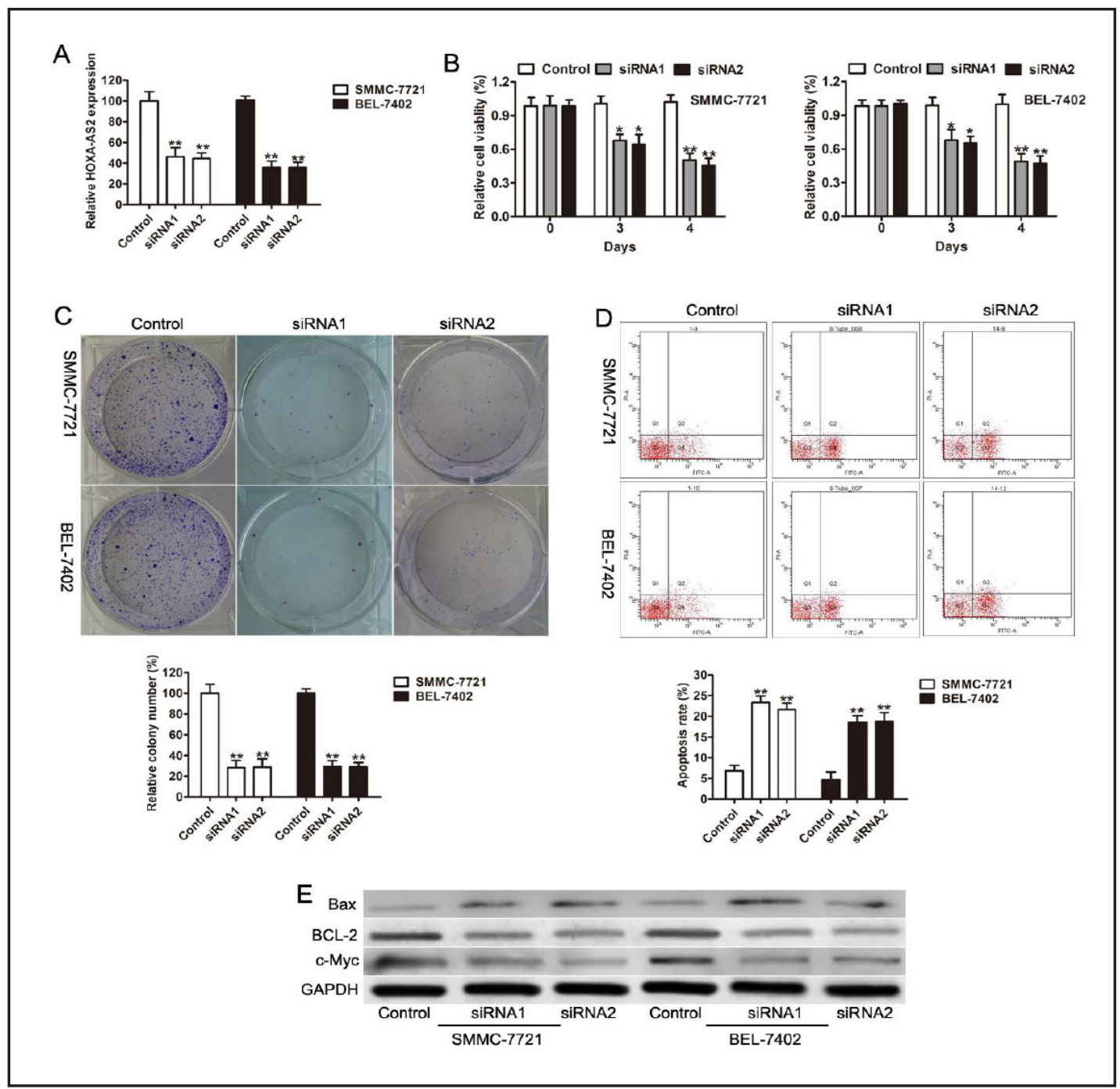

Fig. 3. Effects of HOXA-AS2 knockdown on HCC cells growth. (A) HOXA-AS2 expression level was significantly decreased after transient transfection of HOXA-AS2-specific siRNA in HCC cells as measured by qRT-PCR. (B) Cell proliferation assays were performed to quantify relative cell numbers at the indicated time points. (C) The effect of HOXA-AS2 on the growth of SMMC-7721 and BEL-7402 cells was examined by colony formation assay. (D) The fraction of apoptotic cells was detected in HCC cells transfected with control or siRNA targeting for HOXA-AS2 by flow cytometry. (E) Western blot assays were used to determine the expression of c-Myc, BCL-2 and Bax in SMMC-7721 and BEL-7402 cells. * indicates $\mathrm{P}<0.05$ while ** indicated $\mathrm{P}<0.01$.

qRT-PCR revealed HOXA-AS2 was significantly downregulated by siRNA transfection (Fig. $3 A$ ). Cell proliferation assay revealed that knockdown of HOXA-AS2 significantly inhibited HCC cellular proliferation (Fig. 3B). Clone formation assay also suggested that specific knockdown of HOXA-AS2 in HCC cells lead to a fewer number and a smaller clone than control (Fig. 3C). Then, flow cytometric analysis was performed to further investigate whether the effect of HOXA-AS2 on proliferation of HCC cells by altering apoptosis. The results showed knockdown of HOXA-AS2 could obviously induce cell apoptosis (Fig. 3D). To explore molecular mechanisms by which HOXA-AS2 contributes to the proliferation and apoptosis of HCC cells, the expression of c-Myc, Bcl-2 and Bax was detected by western blot. Depletion of HOXA-AS2 led to a significant decrease of c-Myc and Bcl-2 expression and an increase of Bax (Fig. 3E). These findings indicated that HOXA-AS2 played a positive role in HCC proliferation. 


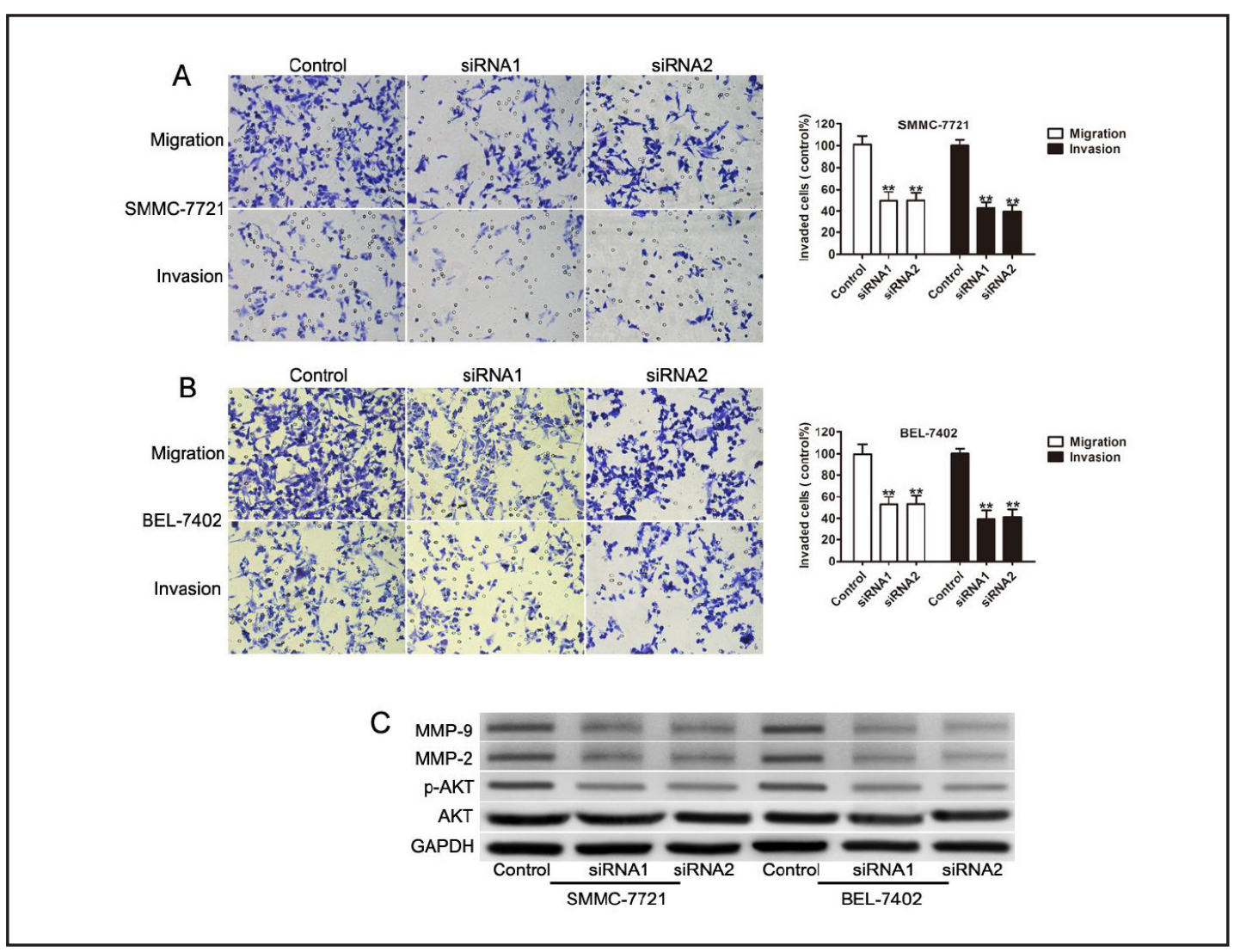

Fig. 4. HCC cell migration and invasion was decreased by silencing of HOXA-AS2. (A and B) Representative images showing staining of SMMC-7721 and BEL-7402 cells on the bottom surface of the filter (upper) and quantitative analysis of the invading cells in 6 random fields (×200). (C) p-AKT (Ser473), MMP-2, MMP-9, and total AKT protein expression was determined by western blot analysis. ${ }^{* *}$ indicated $\mathrm{P}<0.01$.

Knockdown of HOXA-AS2 suppresses the migration and invasion of HCC cells

To further determine the roles of HOXA-AS2 in the motility of HCC cells, transwell migration and matrigel invasion assays were performed in SMMC-7721and BEL-7402 cells. HOXA-AS2 silencing dramatically inhibited cell migration and invasion (Fig. 4A and 4B). To explore the underlying mechanism of HOXA-AS2-mediated inhibition of proliferation and migration of HCC cells, we assessed the expression of p-AKT, MMP-2 and MMP-9, which is thought to be a key process for cancer metastasis. As we expect, western blot analyses showed that knockdown of HOXA-AS2 in HCC cells resulted in a significant decrease of p-AKT, MMP-2 and MMP-9 expression, but not to total AKT. These data suggested that HOXA-AS2-mediated cell migration and invasion through AKT-MMP pathway.

Knockdown of HOXA-AS2 inhibits xenografted tumor growth in vivo

To further verify the effects of HOXA-AS2 on tumorigenesis in vivo, the xenograft tumor model assays were performed by subcutaneously injecting BEL-7402 cells transfected with control or siRNA into the dorsal flank of nude mice. Inhibition of HOXA-AS2 significantly decreased the tumor volume and weight compared with the control (Fig. 5A and B). Tumor growth curve also indicated HOXA-AS2 silencing suppressed tumor growth (Fig. 5C). In addition, to confirm the inhibition of tumor growth was due to the knockdown of HOXAAS2, the expression of HOXA-AS2 was measured in xenograft tumors and our results showed HOXA-AS2 silencing xenograft tumors had lower levels of HOXA-AS2 compared with the control (Fig. 5D). The xenograft tumors were subjected to immunohistochemical staining KARGER 
Fig. 5. HOXA-AS2 silencing inhibited tumor growth in vivo. (A) Representative photographs of tumors from nude mice transplanted with BEL-7402 cells transfected with control or siHOXA-AS2, respectively. (B) The weights of the tumor xenografts. (C) Tumor growth curve were determined by measuring the width and length of the tumors every 7 days. (D) The relative expression of HOXA-AS2 in tumor xenografts tissues from mice. (E) The expression of Ki-67 in the tumor xenografts tissues from mice was detected by IHC. ** indicated $\mathrm{P}<0.01$.
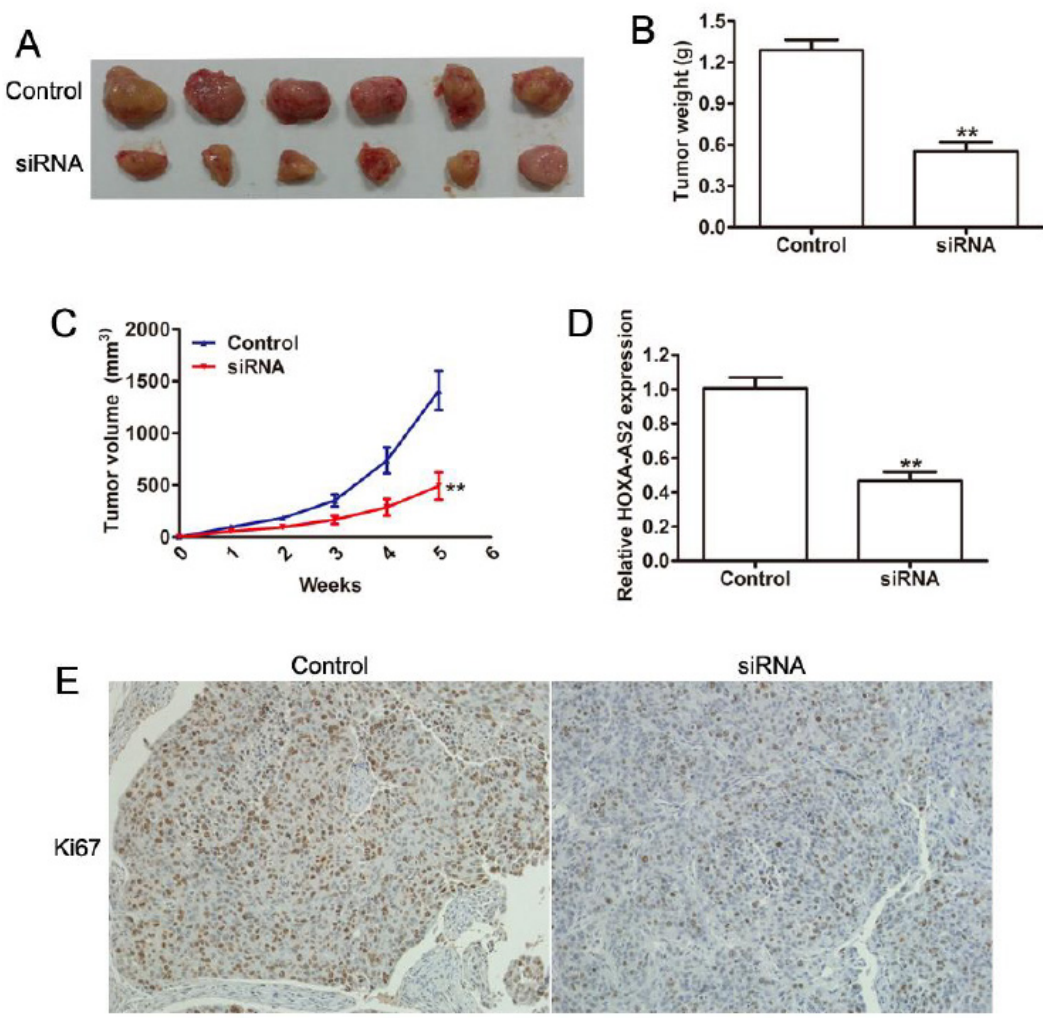

of Ki-67, which indicated the proliferative ability of tumor. Less Ki-67-positive nuclei were observed in HOXA-AS2 silencing xenograft tumors than control xenograft tumors (Fig. 5E). Taken all together, we concluded that HOXA-AS2 silencing inhibited the proliferation of HCC in vitro and in vivo.

\section{Discussion}

Understanding the precise molecular mechanisms underlying HCC tumorigenesis and recurrence is crucial for exploring better therapeutic strategy for patients with HCC. With the development of functional genomics in the past several years, lncRNAs was found to be involved in the gene expression network at various levels, including chromatin modification, transcription, and posttranscriptional processing [9, 11, 23, 24]. Recent reports have shown that aberrant expressions of lncRNAs might alter basic cellular biological processes and contribute to tumorigenesis [25]. Therefore, identification of HCC-associated lncRNAs and their roles may provide a useful strategy for diagnosis and prognosis of patients with HCC.

In this study, we firstly found that HOXA-AS2 was expressed at a higher level in HCC cell lines and HCC tissues. Consisted with previous findings, HOXA-AS2 was upregulated in some kinds of tumor cells, including acute promyelocytic leukemia and gastric cancer $[19,20]$. But little is known about the role of HOXA-AS2 in HCC progression. In the present study, we observed high HOXA-AS2 expression was positively associated with tumor size, TNM stage and short OS, which was consistent with previous findings [20]. In vitro experiments showed that knockdown of HOXA-AS2 could significantly inhibit HCC cell proliferation both in vitro and in vivo. Based on our experiments, inhibition of HOXA-AS2 could be a potential therapy strategy for HCC.

Considering that the clinical stage was majorly concerned about tumor growth and metastasis, we then investigated the effects of HOXA-AS2 on the motility of HCC cells. HOXA- 


\section{Cellular Physiology Cell Physiol Biochem 2016;40:287-296 \begin{tabular}{l|l|l}
\hline DOI: 10.1159/000452545 & $\begin{array}{l}\text { C } 2016 \text { The Author(s). Published by S. Karger AG, Basel } \\
\text { www.karger.com/cpb }\end{array}$ \\
\hline Published online: November 18, 2016 Biochemistry
\end{tabular} \\ Wang et al.: HOXA-AS2 Medicates HCC Proliferation and Migration}

AS2 silencing suppressed cell migration and invasion in HCC cells, which was supported by the downregulation of p-AKT, MMP-2 and MMP-9 expression, which were key molecules in tumor metastasis [26-28]. This finding indicated that high expression of HOXA-AS2 could promote cancer migration and invasiveness ability. Of course, some limitations might be included in our present study. First, only a few of crucial molecules and signaling pathways underlying the enhancement of malignant characteristics were assessed. Second, the present study concerned the roles of HOXA-AS2-silencing in vitro and in vivo. Further investigations were needed to explore the underlying detail mechanisms.

In conclusion, the current study showed that HOXA-AS2 was up-regulated in HCC tissues and high HOXA-AS2 expression predicted poor survival of HCC patients. Furthermore, knocking down of HOXA-AS2 significantly suppressed cell proliferation and invasion, this might provide novel candidate for targeted therapy of HCC patients.

\section{Disclosure Statement}

The authors declare that they have no competing interests.

\section{References}

1 Jemal A, Bray F, Center MM, Ferlay J, Ward E, Forman D: Global cancer statistics. CA Cancer J Clin 2011;61:69-90.

2 Shen Q, Fan J, Yang XR, Tan Y, Zhao W, Xu Y, Wang N, Niu Y, Wu Z, Zhou J, Qiu SJ, Shi YH, Yu B, Tang N, Chu W, Wang M, Wu J, Zhang Z, Yang S, Gu J, Wang H, Qin W: Serum DKK1 as a protein biomarker for the diagnosis of hepatocellular carcinoma: A large-scale, multicentre study. Lancet Oncol 2012;13:817-826.

3 El-Serag HB: Hepatocellular carcinoma: Recent trends in the United States. Gastroenterology 2004;127:S27-S34.

4 El-Serag HB, Rudolph KL: Hepatocellular carcinoma: Epidemiology and molecular carcinogenesis. Gastroenterology 2007;132:2557-2576.

$5 \quad$ Ponting CP, Oliver PL, Reik W: Evolution and functions of long noncoding RNAs. Cell 2009;136:629-641.

6 Kung JT, Colognori D, Lee JT: Long noncoding RNAs: Past, present, and future. Genetics 2013;193:651-669.

7 Gutschner T, Diederichs S: The hallmarks of cancer: A long non-coding RNA point of view. RNA Biol 2012;9:703-719.

8 Zhou X, Ye F, Yin C, Zhuang Y, Yue G, Zhang G: The interaction between MiR-141 and lncRNA-H19 in regulating cell proliferation and migration in gastric cancer. Cell Physiol Biochem 2015;36:1440-1452.

9 Li J, Wang X, Tang J, Jiang R, Zhang W, Ji J, Sun B: HULC and linc00152 act as novel biomarkers in predicting diagnosis of hepatocellular carcinoma. Cell Physiol Biochem 2015;37:687-696.

10 Prensner JR, Chinnaiyan AM: The emergence of lncRNAs in cancer biology. Cancer Discov 2011;1:391-407.

11 Guttman M, Rinn JL: Modular regulatory principles of large non-coding RNAs. Nature 2012;482:339-346.

12 Gu M, Zheng A, Tu W, Zhao J, Li L, Li M, Han S, Hu X, Zhu J, Pan Y, Xu J, Yu Z: Circulating LncRNAs as novel, Non-Invasive biomarkers for prenatal detection of fetal congenital heart defects. Cell Physiol Biochem 2016;38:1459-1471.

13 Tang J, Zhuo H, Zhang X, Jiang R, Ji J, Deng L, Qian X, Zhang F, Sun B: A novel biomarker Linc00974 interacting with KRT19 promotes proliferation and metastasis in hepatocellular carcinoma. Cell Death Dis 2014;5:e1549.

14 Gupta RA, Shah N, Wang KC, Kim J, Horlings HM, Wong DJ, Tsai MC, Hung T, Argani P, Rinn JL, Wang Y, Brzoska P, Kong B, Li R, West RB, van de Vijver MJ, Sukumar S, Chang HY: Long non-coding RNA HOTAIR reprograms chromatin state to promote cancer metastasis. Nature 2010;464:1071-1076.

15 Kogo R, Shimamura T, Mimori K, Kawahara K, Imoto S, Sudo T, Tanaka F, Shibata K, Suzuki A, Komune S, Miyano S, Mori M: Long noncoding RNA HOTAIR regulates polycomb-dependent chromatin modification and is associated with poor prognosis in colorectal cancers. Cancer Res 2011;71:6320-6326.

16 Sun X, Du P, Yuan W, Du Z, Yu M, Yu X, Hu T: Long non-coding RNA HOTAIR regulates cyclin J via inhibition of microRNA-205 expression in bladder cancer. Cell Death Dis 2015;6:e1907. 


\section{Cellular Physiology Cell Physiol Biochem 2016;40:287-296 \begin{tabular}{ll|l} 
DOI: 10.1159/000452545 & $\begin{array}{l}\text { O 2016 The Author(s). Published by S. Karger AG, Basel } \\
\text { www.karger.com/cpb }\end{array}$
\end{tabular} \\ Wang et al.: HOXA-AS2 Medicates HCC Proliferation and Migration}

17 Bhan A, Mandal SS: LncRNA HOTAIR: A master regulator of chromatin dynamics and cancer. Biochim Biophys Acta 2015;1856:151-164.

18 Luo G, Wang M, Wu X, Tao D, Xiao X, Wang L, Min F, Zeng F, Jiang G: Long Non-Coding RNA MEG3 inhibits cell proliferation and induces apoptosis in prostate cancer. Cell Physiol Biochem 2015;37:2209-2220.

19 Zhao H, Zhang X, Frazao JB, Condino-Neto A, Newburger PE: HOX antisense lincRNA HOXA-AS2 is an apoptosis repressor in all trans retinoic acid treated NB4 promyelocytic leukemia cells. J Cell Biochem 2013;114:2375-2383.

20 Xie M, Sun M, Zhu YN, Xia R, Liu YW, Ding J, Ma HW, He XZ, Zhang ZH, Liu ZJ, Liu XH, De W: Long noncoding RNA HOXA-AS2 promotes gastric cancer proliferation by epigenetically silencing P21/PLK3/DDIT3 expression. Oncotarget 2015;6:33587-33601.

21 Zhang ZZ, Hua R, Zhang JF, Zhao WY, Zhao EH, Tu L, Wang CJ, Cao H, Zhang ZG: TEM7 (PLXDC1), a key prognostic predictor for resectable gastric cancer, promotes cancer cell migration and invasion. Am J Cancer Res 2015;5:772-781.

22 Ma Y, Yue Y, Pan M, Sun J, Chu J, Lin X, Xu W, Feng L, Chen Y, Chen D, Shin VY, Wang X, Jin H: Histone deacetylase 3 inhibits new tumor suppressor gene DTWD1 in gastric cancer. Am J Cancer Res 2015;5:663673.

23 Wang KC, Chang HY: Molecular mechanisms of long noncoding RNAs. Mol Cell 2011;43:904-914.

24 Zhang F, Zhang L, Zhang C: Long noncoding RNAs and tumorigenesis: Genetic associations, molecular mechanisms, and therapeutic strategies. Tumour Biol 2015

25 Zhao J, Liu Y, Huang G, Cui P, Zhang W, Zhang Y: Long non-coding RNAs in gastric cancer: Versatile mechanisms and potential for clinical translation. Am J Cancer Res 2015;5:907-927.

26 Yoo YA, Kang MH, Lee HJ, Kim BH, Park JK, Kim HK, Kim JS, Oh SC: Sonic hedgehog pathway promotes metastasis and lymphangiogenesis via activation of Akt, EMT, and MMP-9 pathway in gastric cancer. Cancer Res 2011;71:7061-7070.

27 Kenny HA, Lengyel E: MMP-2 functions as an early response protein in ovarian cancer metastasis. Cell Cycle 2009;8:683-688.

28 Melnikova VO, Mourad-Zeidan AA, Lev DC, Bar-Eli M: Platelet-activating factor mediates MMP2 expression and activation via phosphorylation of cAMP-response element-binding protein and contributes to melanoma metastasis. J Biol Chem 2006;281:2911-2922. 